\title{
ANALYSIS OF GENE ACTION IN THE CONTROL OF BODY WEIGHT AND TAIL LENGTH IN THE MOUSE
}

\author{
J. R. MORTON \\ Department of Agricultural Science and Applied Biology, University of Cambridge
}

Received 1.i.70

\section{INTRODUCTION}

A METHOD has been published (Morton and O'Donald, 1962) for the analysis of gene action from crosses of inbred animals, which placed greater emphasis on the estimation of interactions between pairs of loci than the earlier formulations of Mather and Hayman (e.g. Mather, 1949; Hayman, 1958), from which it was derived. We believed that interaction would be of greater importance in naturally outbreeding organisms than in the normally selffertilising plants for which the Hayman-Mather formulae were primarily intended. A number of continuously varying traits in mice and chicken were analysed using this method, although the results are not generally available (Morton, 1963). In a brief summary of the results (Morton, 1964), it was concluded that those traits more nearly associated with fitness showed large dominance deviations acting in the opposite direction to large or even larger dominance $\times$ dominance interactions. In the present paper the analysis has been improved and extended, and the conclusions of Morton (1964) have been correlated with recent advances in genetic theory, particularly the related mathematical models of Sved, Reed and Bodmer (1967), King (1967) and Maynard Smith (1968), all of which involve forms of epistasis. For example King (loc. cit.) predicts that, where the average decrement in fitness associated with each substitution is the standard of measurement, considerable negative synergism should exist over the whole range of possible genotypes. The opposition of dominance and dominance interaction of Morton (1964) and the negative synergism of King (1967) are similar to the duplicate interaction found in Drosophila by Breese and Mather (1960). This was interpreted by Breese and Mather as a further development of the evolution of dominance, which results in an overall increase in the fitness of the population (Mather, 1966). Such epistasis is measurable with suitable experimental design. For example, associations may be sought between groups of polymorphic loci and components of fitness, as we have already done (Morton et al., 1965; Gilmour and Morton, 1970). Another approach is to analyse the forms of epistasis in the control of continuously varying traits over a wide range of genotypes, to see which forms are associated with traits determining fitness; in this context the observation of balancing dominance and dominance $\times$ dominance interaction effects (Morton, 1964) is of obvious significance.

This paper presents an analysis of the forms of gene action affecting the characters body weight and tail length of mice at 2, 4 and 6 weeks of age.

\section{Materials and data}

Since the use of large and small strains derived by selection might bias the results in favour of additive gene action, it was decided to use two highly 
inbred lines which happened to differ considerably in body size. It was presumed that these would be largely homozygous and that the genes for body size would be largely associated rather than dispersed (Jinks and Jones, 1958). The $C B A / C a g$ and $A / C a g$ standard inbred lines (Staats, 1968) were thus chosen. At six weeks of age the $C B A$ mice exceeded the $A$ in body weight by 17.7 per cent. in males and 12.2 per cent. in females. The mice were bred and reared at constant temperature of $25^{\circ} \mathrm{C}$. (range $24^{\circ}-27^{\circ} \mathrm{C}$.). Temperature control was of particular importance in view of the findings of Harrison et al. (1959) that the tail of the mouse is a heat regulatory organ and that its length depends largely on the temperature at which the animal is reared. The mice were fed ad libitum on the standard Blue Cross diet 41B.

All mice were bred by single-pair matings. New matings of the two pure lines were made continuously throughout the experiment. No time trends in weight or tail length were observed. At least eight successful matings were made for the reciprocal first crosses and for each filial generation derived from them up to the $\mathrm{F}_{4}$, and at least two successful matings of each of the eight possible back-crosses and sibbed back-crosses. All offspring produced during the active breeding life of these matings are included in the analysis (except those born on a Sunday), since earlier work had suggested the effects of parity and litter size are negligible. Thus Falconer (1947) and Butler (1952) found no effect of parity on body weight. Butler as well as Chai (1957) found no effect of litter size on body weight, although Falconer (1947) found a very small reduction in weight as the litter size increased beyond four, and Biggers et al. (1958) noted that a small effect at 2 weeks had wholly disappeared by 4 weeks of age. It was also felt that attempts to equalise litter size might introduce more error than they removed if the dams producing the larger litters had a greater inherent ability to rear them. Subsequently Jinks and Broadhurst (1963) noted such a tendency in rats where the strain of dams producing the largest litters also produced the largest individual pups.

At 2, 4 (weaning) and 6 weeks of age the mice were weighed to the nearest $0.1 \mathrm{gm}$. and their tails measured to the nearest $\mathrm{mm}$., the length of the tail when straightened but not stretched being taken from the end of the body hair to the tip. Falconer (1947) found that the error variance of 12-day minus 5-day weight was so much greater than that of 12-day weight alone that the latter was preferable as a measure of juvenile growth in mice. Analysis is therefore made of the 2-, 4- and 6-week measurements themselves and not of the differences between them.

\section{Preliminary analyses}

\section{(i) Scaling}

Although the choice of a scale which correctly reflects the form of action of the genes is less crucial in an analysis by generation means, such as used here, than in one by generation variances (Gilbert, 1961), a wholly inappropriate choice could still alter the relative values of the estimates.

The scaling methods of Mather (1949) are unsuitable since they are based on the minimisation of epistasis, whereas we wish to make realistic estimates of the various epistatic components. Furthermore, if epistasis is present, a scale which removes it will also distort the relative magnitudes of the additive and dominance effects. 
Morton and O'Donald (1962) proposed a method of deriving an appropriate scale from the data, for metric traits showing a sex differential. It was based on the premise that genes associated with maleness enhance the normal mode of action of the genes controlling the trait. The correct scale will be that which will convert to linearity the curvilinear regression of sex differential against female or mid-sex value. The mid-sex value is preferred since it will lessen the effects of fortuitous association of low female values with large sex differentials. In these data the sex differential is very small and of variable sign in 2-week weight and in 2- and 4-week tail length. The method was therefore applied only to 6-week tail length and 4- and 6week body weight. No significant regression was found for tail length. The regressions for 4- and 6-week weight were significantly linear $(+0.22$ \pm 0.06 and $+0.38 \pm 0.08$, respectively) with no significant improvement of fit from fitting second-order terms $\left(F_{1,21} 1 \cdot 72\right.$ and $\left.0 \cdot 12\right)$. However, the linear regressions account for only 51 per cent. and 35 per cent. of the variability respectively. When the data were plotted there were wide and general scatters which could not be accounted for by any simple polynomial. The reasons for this scatter and hence the failure of the method will become clear in the results.

Of various other methods of testing the suitability of a scale, the absence of kurtosis in the distribution in the $F_{1}$ populations (Sewall Wright, 1952) was that favoured by Morton and O'Donald (1962). The present data satisfy this criterion without transformation, so that from the evidence so far there appears no reason for the use of any transformation. However, Falconer (1953) argued that the tendency in body weight of mice for the variances, or more strictly the standard deviations, to increase with the mean values is evidence for multiplicative gene action requiring transformation to a logarithmic scale. Similar observations and conclusions have been reached by other workers (e.g. Chai, 1956; Biggers et al., 1958). In the present data the variances at 4 weeks, the period of fastest growth, are inflated, but whereas the variance of body weight is much greater at 6 than at 2 weeks, the variances of tail length are similar at these ages. This suggests multiplicative gene action on weight but additive action on tail length.

We may also compare the means and standard deviations of generations of widely different means and similar expected genetic variances. The genetic variances of the parental and $F_{1}$ generations should be zero; but in these data the variances in the $A$ line appear inflated, possibly as a result of genetic imbalance, and the means of the $C B A$ and $\mathrm{F}_{1}$ generations do not differ greatly. However, the back-cross generations, having similar although not identical expected genetic variances, do show a wide range of means.

The regressions of standard deviation on mean for each of the 8 backcross generations are small (table 1), but all are negative for tail length and all positive for body weight.

The negative regressions for tail length at first suggest that a transformation such as anti-log might be appropriate, but this is rejected as inherently unreasonable. If they arise from gene effects, they indicate some form of epistasis, such as duplicate interaction, which we are more interested to investigate than to remove by such arbitrary scaling. On the other hand the positive values for body weight may arise from the kind of interaction which is inherent in the anatomy and physiology of the animal rather than from a genetic effect. As Williams (1959) showed for the tomato, if inheritance is 
additive on linear measurement it will necessarily be multiplicative on measurements of mass. This is the type of effect which should be eliminated by transformation. With true multiplicative action the regression of standard deviation on mean is expected to have a value of +1 . That the values for mouse body weights are clearly less than unity could in part result from the types of interaction indicated by the negative regressions for tail length. But also, while genetic effects on body weight may be multiplicative, error effects - particularly those related to the physical weighing of the animalswill be normally distributed on the additive scale, as is shown by the normal distributions of the $F_{1}$ populations on the additive scale. This will mean that the genetic parameters may be best estimated on a log scale but their standard errors on the additive scale.

\section{TABLE 1}

Coefficients of the regression of standard deviations on means of the 8 back-cross generations

$\begin{array}{lccc} & 2 \text { weeks } & 4 \text { weeks } & 6 \text { weeks } \\ \text { Tail length } & -0.03 & -0.07 & -0.03 \\ & -0.09 & -0.13 & -0.18 \\ \text { Body weight } & & & \\ & +0.17 & +0.25 & +0.01 \\ & +0.15 & +0.10 & +0.09\end{array}$

Because of these difficulties, it was decided to analyse the tail length measurements on the additive scale only, but the body weights on both additive and $\log$ scales. It will be seen that this apparently unsatisfactory compromise is justified by the close agreement between the results on the two scales, as predicted for an analysis by mean values (Gilbert, 1961; Morton and O'Donald, 1962). Following Sewall Wright (1952), the means and variances were converted to logs by the expressions

and

$$
\begin{aligned}
\overline{\log _{10} x} & =\log _{10} \bar{x}-\frac{1}{2} \log _{10}\left(1+C^{2}\right) \\
s^{2} \log _{10} x & =0.43431 \log _{10}\left(1+C^{2}\right)
\end{aligned}
$$

where $C^{2}=$ the coefficient of variabilty of $x$.

\section{(ii) Association and dispersion}

Although the method of means analysis reduces errors due to unequal effects of different genes or to inappropriateness of scale, it only correctly estimates all genetic parameters if the genes are wholly associated. Lack of association does not affect the estimates of dominance and dominance interaction effects, in which we are primarily interested, but, to the extent that the smaller line contains some alleles for larger size, the estimates of additive effects will be too small, and those of additive interactions distorted (Jinks and Jones, 1958). The lines providing the present data were chosen to differ markedly in size as an attempt to overcome this problem, but it is none the less necessary to consider how far dispersion (lack of association) may have affected the various additive estimates.

A simple approximate method of estimating the degree of dispersion follows from the familiar observation that the distribution of measurements made in the $F_{2}$ generation commonly exceeds the equivalent distributions 
of the parental generations. When the distributions of these three generations are scaled by working in standard deviations and superimposed by placing the mean of the $\mathrm{F}_{2}$ midway between those of the pure lines, we expect that, if the genes are fully associated, none of the distribution of the $F_{2}$ generation should fall outside those of the parental generations. If on the other hand the genes are fully dispersed, the means and modes of the three distributions should be coincident but that of the $\mathrm{F}_{2}$ should at all points lie outside those of the parent generations. In intermediate cases the proportion of the $F_{2}$ distribution lying beyond the intersection of the $F_{2}$ and pure distributions gives an approximate estimate of the degree of dispersion. The existence of overdominance or of certain forms of epistasis could cause an overestimate of the degree of dispersion using this method. But we are here concerned if possible to demonstrate the absence of dispersion, so that any such error will be conservative. Further, to make the test as conservative as possible the more variable of the two $F_{2} s$ from reciprocal $F_{1} s$ was used in each case in making these estimates (table 2).

TABLE 2

\begin{tabular}{|c|c|c|c|c|}
\hline \multicolumn{5}{|c|}{ Approximate estimates of degree of genetic dispersion (\%) } \\
\hline & & Tail length & Weight & Log weight \\
\hline 2 weeks & $\begin{array}{l}9 \% \\
\text { वे० }\end{array}$ & $\begin{array}{l}39 \\
20\end{array}$ & $\begin{array}{l}91 \\
88\end{array}$ & $\begin{array}{l}43 \\
40\end{array}$ \\
\hline 4 weeks & 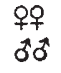 & $\begin{array}{l}\ll 1 \\
\ll 1\end{array}$ & $\begin{array}{r}14 \\
8\end{array}$ & $\begin{array}{l}0 \\
0\end{array}$ \\
\hline 6 weeks & $\begin{array}{l}\text { 99 } \\
\text { उٓరే }\end{array}$ & $\begin{array}{l}\ll 1 \\
\ll 1\end{array}$ & $\begin{array}{l}4 \\
2\end{array}$ & $\begin{array}{l}<1 \\
<1\end{array}$ \\
\hline
\end{tabular}

From the results it is clear that genetic association is nearly complete in the characters measured at 4 and 6 weeks of age, but that at 2 weeks there is considerable dispersion. This will need to be recalled when the estimates of the additive effects at 2 weeks are considered.

\section{Main analysis}

There were available for analysis the two parental lines, two reciprocal $\mathrm{F}_{1} \mathrm{~s}$, and the subsequent filial generations up to the $\mathrm{F}_{4}$ of each, the eight possible backcrosses and sibbed backcrosses, a total of 26 generations. Measurements were made on more than 3500 mice in all.

The mean of each generation was equated to the genetic parameters as defined in Morton and O'Donald (1962). In addition, parameters were set for maternal and sex-linked effects. Clearly every generation could have a different maternal effect, but the number that can be estimated is limited by the number of generation means available and of genetic parameters to be estimated. It was decided to set parameters for each inbred line and for the $F_{1}$, representing the genotypic extremes both from the point of view of genetic origin and of that of inbreeding versus crossbreeding, since heterosis was known to be an important component of maternal effect on body size (Brumby, 1960). As it appeared from inspection that the reciprocal $F_{1}$ s had different maternal effects, a separate parameter was set for each. These four generations of dams for which maternal effect parameters were 
set were the dams of 14 of the 26 generations bred. The remaining generations of dams, back-crosses, $\mathrm{F}_{2} \mathrm{~s}$ and $\mathrm{F}_{3} \mathrm{~s}$, are of less extreme genotypes, and their maternal effects are assumed to differ negligibly from one another. Thus effectively the maternal effects of the pure lines and $F_{1} s$ were estimated as deviations from those of the remainder. The X-linked parameter was defined by giving the value of +1 to a $C B A \mathrm{X}$ chromosome and -1 to an $A \mathrm{X}$ chromosome, thus allowing for the fact that one random $\mathrm{X}$ chromosome is inactivated in the female mammal (Lyon, 1962). The $\mathrm{Y}$ chromosome effects were estimated by giving the $\mathrm{Y}$ chromosome of $C B A$ the value of +1 and that of $A$ zero, and omitting the parameter entirely in the female data.

In this basic model, hereafter called the digenic model, there were thus 12 parameters to be estimated, namely, $m=$ notional mean, and deviations from this mean as follows:

$$
\begin{aligned}
& d=\text { additive } \\
& h=\text { dominance } \\
& i=\text { additive } \times \text { additive } \\
& j=\text { additive } \times \text { dominance } \\
& l=\text { dominance } \times \text { dominance } \\
& r=A \text { dam maternal } \\
& n=C B A \text { dam maternal } \\
& p=\left(A \& \times C B A{ }^{\star}\right) \mathrm{F}_{1} \text { dam maternal } \\
& q=\left(C B A \text { o } \times A{ }^{\star}\right) \mathrm{F}_{1} \text { dam maternal } \\
& x=\mathrm{X} \text { chromosomal } \\
& y=\mathrm{Y} \text { chromosomal }
\end{aligned}
$$

As two pairs of sibbed back-crosses had identical expectations of these parameters, there were effectively 24 generation means to estimate them.

The estimation of the parameters followed in general the method introduced by Cavalli (1952). However, from inspection of the data it appeared that certain of the generations means based on the smallest number of individuals had the smallest variances, so that the latter may not have been well estimated. Therefore the reciprocal of the variance attributable to the mean was replaced by the ratio of the number of individuals to the largest observed variance within a generation for that sex and character; and these were used as weights for the 24 equations to estimate the parameters. The resultant solutions will then not be strictly maximum likelihood, but they will eliminate the risk of falsely attributing significance to the estimates due to underestimation of their error variances. The equations were solved separately for each sex by standard least squares techniques using a computer.

The method provides standard errors for the estimates of the parameters simply, for if as a result of the least squares solution, the 12 parameters, $e_{j}$, are related to the 24 generation means, $\bar{x}_{i}$, by the coefficients $c_{j i}$, thus

$$
e_{j}=\sum_{i} c_{j i} \bar{x}_{i}
$$

their standard errors, $s_{j}$, are given by

$$
s_{j}=\sum_{i} c^{2}{ }_{j i} V_{i},
$$

where $V_{i}$ are the estimated error variances of the means, $\bar{x}_{i}$, that is, the reciprocals of the weights applied to them. The goodness of fit of the whole 
model to the data was tested by

$$
\chi^{2}=\sum_{i} \frac{1}{V_{i}}\left(\bar{x}_{i}-E \bar{x}_{i}\right),
$$

where $E \bar{x}_{i}$ are the expectations of the generation means from the estimated parameters, $e_{j}$, and the degrees of freedom attributable to the $\chi^{2}$ are the difference between the numbers of generations and parameters.

The initial fit of the model to the data was very poor, as shown by the $\chi^{2}$ values in the first two rows of table 3. It was therefore decided to fit models that allow of more complex interactions. In the $\mathrm{F} \infty$ type of model the coefficients of the parameters for interaction between two or three loci are obtained as the second or third terms of the polynomial expansion of the coefficients of the parameters for the loci considered singly. Extension to more complex models, indeed beyond those involving three-locus interactions, can thus be simply derived from the basic autosomal coefficients (Morton and O'Donald, 1962) and those set for sex-linkage, etc. Two such further models were tried, a trigenic model, in which interactions between three autosomal loci are fitted and analogous to the trigenic model of Jinks and Perkins (1969) and a digenic model with sex-linked interaction, in which parameters were set for interactions between loci on the sex chromosomes and loci on the autosomes, considering one of each type of locus at a time.

Although the fit of the male data was better to the trigenic model than the digenic (table 3, row 4), that of the female data was if anything worse (table 3, row 3). Furthermore, various of the estimates of the parameters seemed impossibly large, in a way and for reasons which will be considered later. The results with the digenic model with sex-linked interaction, although marginally improved in fit, were essentially similar to those with the trigenic model (table 3 , rows 5 and 6 ).

Since there appeared to be a large difference in the maternal effects of the reciprocal $F_{1}$ generations, and since Brumby (1960) had reported cytoplasmic effects in the inheritance of body weight in mice, a parameter for the effect of the $A$ strain cytoplasm relatife to that of $C B A$ was introduced into both digenic and trigenic models. The fit was not however improved.

The individual contributions of each generation to the total $\chi^{2}$ for goodness of fit to the digenic model were then inspected. It was found that of the 11 individual contributions greater than 10,8 were attributable to the generation $\mathrm{B}_{21}\left(=C B A\right.$ 웅 $\times\left(\mathrm{A}_{+} \times C B A_{0}^{\top}\right) \mathrm{F}_{1} 0^{\star}$ back-cross $)$. In each of these 8 cases, and in a further 6 in which the contribution of this generation to $\chi^{2}$ was greater than 5 , the mean of $\mathrm{B}_{21}$ was lower than could be accounted for by the model and the remainder of the data. Inspection of the original data sheets showed that the number of mice of generation $B_{21}$ dying before 6 weeks of age was unusually large. (Casualties were in general very rare.) It seems then reasonable to suppose that the cause of death in these mice also stunted the growth of many of those of this generation which survived. The removal of $B_{21}$ from the data permitted a much improved fit to the model in most cases (table 3 , rows 7 and 8 ). The estimates of the parameters derived from the digenic model with the reduced data are shown in table 4 .

Since the fit of the digenic model was still less than perfect, both the more complex models were again applied but to the reduced data. For the trigenic model there was an apparent improvement of fit in the males, 


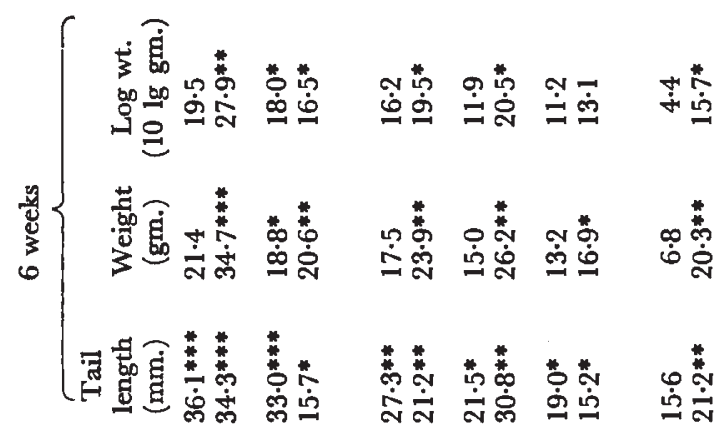

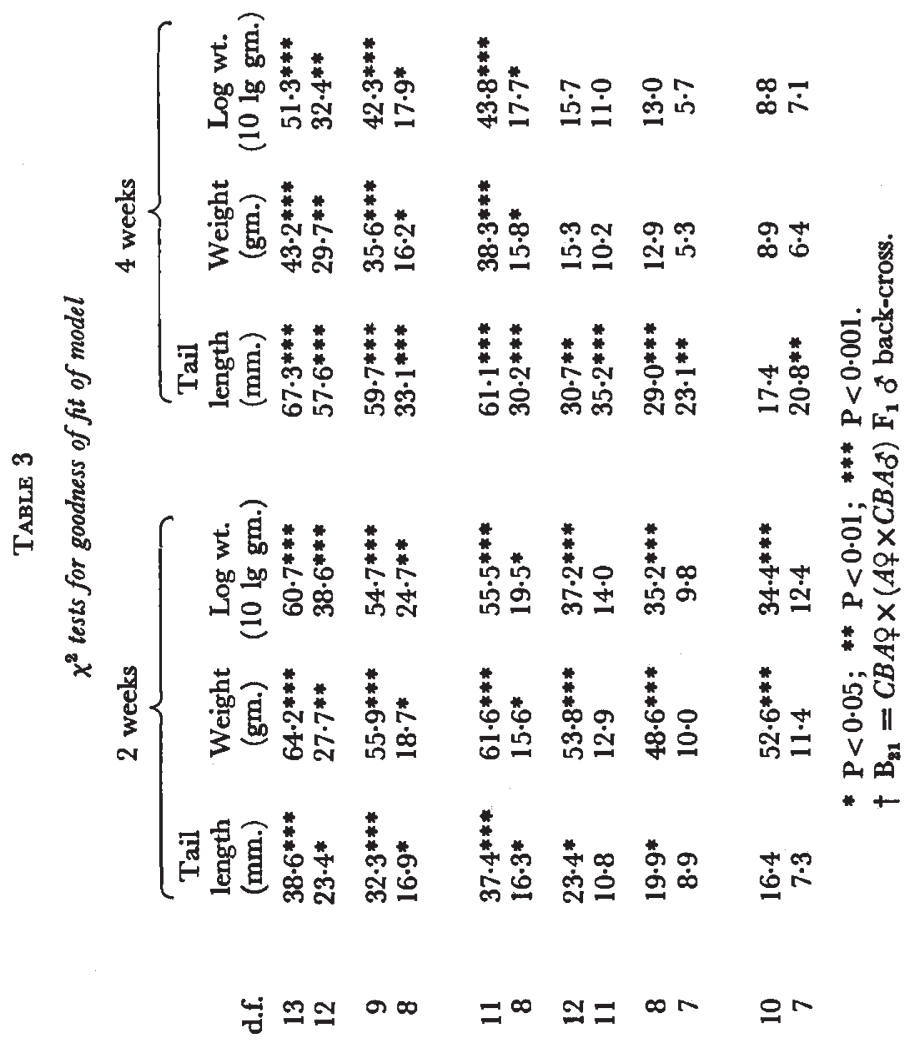

ot $\begin{array}{lll}r_{0} \\ \text { ot }\end{array}$

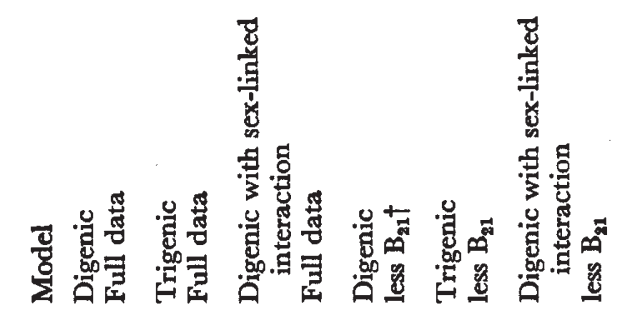


for 4-week tail length and for all measurements at 6 weeks of age. The estimates of the parameters, however, demonstrated the same peculiarities that were briefly noted for those derived by the trigenic model from the full data. The parameters fitted for the notional mean rose well above the actual mean of the data, and several of the genetic parameters, particularly but not exclusively those for dominance and dominance interaction, were several orders larger still, up to more than seven times the notional mean.

TABLE 4

Estimates of the congenital parameters for the digenic model less $B_{21}$

\begin{tabular}{|c|c|c|c|c|c|c|c|c|c|}
\hline & & 2 weeks & & & 4 weeks & & & 6 weeks & \\
\hline & $\begin{array}{l}\text { Tail } \\
\text { length } \\
\text { (mm.) }\end{array}$ & $\begin{array}{l}\text { Weight } \\
\text { (gm.) }\end{array}$ & $\begin{array}{c}\text { Log } \\
\text { weight } \\
\text { (10 lg gm.) }\end{array}$ & $\begin{array}{c}\text { Tail } \\
\text { length } \\
\text { (mm.) }\end{array}$ & $\begin{array}{l}\text { Weight } \\
\text { (gm.) }\end{array}$ & $\begin{array}{c}\text { Log } \\
\text { weight } \\
\text { (10 lg gm.) }\end{array}$ & $\begin{array}{c}\text { Tail } \\
\text { length } \\
\text { (mm.) }\end{array}$ & $\begin{array}{l}\text { Weight } \\
\text { (gm.) }\end{array}$ & $\begin{array}{c}\text { Log } \\
\text { weight } \\
(10 \lg \text { gm. })\end{array}$ \\
\hline $\begin{array}{l}m \\
d \\
h \\
i \\
j \\
l \\
m \\
n \\
p \\
q \\
x\end{array}$ & $\begin{array}{l}42 \cdot 0 \\
-2 \cdot 7 * * * \\
-4 \cdot 0 \\
-3 \cdot 3^{*} \\
+0 \cdot 1 \\
+2 \cdot 8 \\
-2 \cdot 1^{* *} \\
+1 \cdot 0 \\
+2 \cdot 1 * * * \\
+0 \cdot 9 \\
+1 \cdot 9^{* *}\end{array}$ & $\begin{array}{l}6.1 \\
-0.8^{* * * *} \\
+0.3 \\
+0.5 \\
-0.1 \\
+0.5 \\
-1.0^{* * *} \\
-0.3 \\
+0.6^{* * *} \\
+0.4^{* *} \\
+0.5^{* * *}\end{array}$ & $\begin{array}{l}7.9 \\
-0.5^{* * *} \\
+0.1 \\
+0.3 \\
+0.0 \\
+0.3 \\
-0.7 * * * \\
-0.1 \\
+0.3^{* * *} \\
+0.3^{* *} \\
+0.2^{*}\end{array}$ & $\begin{array}{l}60.3 \\
+1.7 * \\
+7.5 \\
-1.7 \\
+0.1 \\
-3.9 \\
-1.6 \\
+0.0 \\
+2.7 * * * \\
+1.7 * * \\
+0.3\end{array}$ & $\begin{array}{l}11.2 \\
-0.2 \\
+4.4 * * \\
+0.6 \\
-0.3 \\
-1.3 \\
-1.3^{* * *} \\
-0.2 \\
+0.7 * * \\
+1.0 * * * \\
-0.1\end{array}$ & $\begin{array}{l}10.4 \\
-0.0 \\
+1.8^{* *} \\
+0.2 \\
-0.1 \\
-0.6 \\
-0.4 * * * \\
-0.1 \\
+0.2^{* *} \\
+0.3 * * * \\
-0.0\end{array}$ & $\begin{array}{l}71.2 \\
+1.9 * * \\
+7.0^{*} \\
-1.6 \\
-1.2 \\
-2.8 \\
-1.3 \\
-0.1 \\
+1.4 * \\
+0.2 \\
+1.4 *\end{array}$ & $\begin{array}{l}15.3 \\
-0.5 \\
+4 \cdot 1^{* *} \\
+1.6^{* *} \\
-1.3^{* * *} \\
-0.3 \\
-1 \cdot 7^{* * *} \\
-0.8^{*} \\
+0.9^{* * *} \\
+0.2 \\
+0.9^{* * *}\end{array}$ & $\begin{array}{l}11.8 \\
-0.1 \\
+1 \cdot 1^{* *} \\
+0.4 * * \\
-0.3^{* * *} \\
-0.1 \\
-0.4^{* * *} \\
-0.2^{*} \\
+0.2^{* * *} \\
+0.0 \\
+0.2 * * *\end{array}$ \\
\hline $\begin{array}{l}m \\
d \\
h \\
i \\
j \\
l \\
m \\
n \\
p \\
q \\
x \\
v\end{array}$ & $\begin{array}{l}40.2 \\
+0.8 \\
+2.8 \\
-0.9 \\
+0.2 \\
-1.3 \\
-2.5^{*} \\
+1.3 \\
+1.5^{*} \\
+1.0 \\
-1.1 \\
-1.5^{*}\end{array}$ & $\begin{array}{l}6.0 \\
-0.2 \\
+1.2 \\
+0.8^{*} \\
+0.2 \\
+0.1 \\
-1.3^{* * *} \\
-0.5 \\
+0.4^{* *} \\
+0.5^{* * *} \\
-0.3 \\
-0.3\end{array}$ & $\begin{array}{l}7.7 \\
-0.1 \\
+0.9 \\
+0.5^{*} \\
+0.2 \\
-0.1 \\
-0.9^{* * *} \\
-0.0 \\
+0.2^{* *} \\
+0.3^{* * *} \\
-0.2 \\
-0.2\end{array}$ & $\begin{array}{l}60.5 \\
+2.8^{* *} \\
+9.5^{*} \\
-0.9 \\
+0.6 \\
-5.2 \\
-2.4^{*} \\
+1.0 \\
+1.5^{*} \\
+2.0^{* *} \\
-1.6 \\
-0.9\end{array}$ & $\begin{array}{l}11.7 \\
-0.4 \\
+6.0 * * \\
+1.3 \\
+0.0 \\
-1.7 \\
-1.4 * * \\
-0.3 \\
+0.6 \\
+1.4 * * * \\
+0.3 \\
-0.2\end{array}$ & $\begin{array}{l}10.7 \\
-0.1 \\
+2.0 * * \\
+0.3 \\
-0.0 \\
-0.7 \\
-0.4 * \\
-0.1 \\
+0.2 \\
+0.4 * * * \\
+0.1 \\
-0.1\end{array}$ & $\begin{array}{c}72 \cdot 5 \\
+6 \cdot 0^{* * *} \\
+10 \cdot 0^{* *} \\
-0.3 \\
-1 \cdot 1 \\
-4 \cdot 1 \\
-2 \cdot 1 * \\
-0 \cdot 1 \\
+0 \cdot 8 \\
-0.1 \\
-2 \cdot 1 * * \\
-1 \cdot 8^{* *}\end{array}$ & $\begin{array}{l}18 \cdot 0 \\
+1.7 * * * \\
+7 \cdot 6^{* * *} \\
+2 \cdot 0^{* *} \\
-1 \cdot 1^{* *} \\
-2.4 \\
-1 \cdot 8^{* * *} \\
-0.6 \\
+0.8^{* *} \\
+0.6^{*} \\
-0.4 \\
-0.9 *\end{array}$ & $\begin{array}{l}12.5 \\
+0.4^{* * *} \\
+1.7^{* * *} \\
+0.4^{*} \\
-0.3^{* *} \\
-0.6 \\
-0.4^{*} \\
-0.1 \\
+0.2^{*} \\
+0.1 \\
-0.1 \\
-0.2^{*}\end{array}$ \\
\hline
\end{tabular}

For definitions of congenital parameters, see text.

The cause of these unsatisfactory results probably lies in the correlations that can arise between estimates as noted by Hayman (1958). Where the design of the experiment, as here, lies largely in the vertical, that is derives largely from continued sib-mating, correlations will arise of additive with additive interaction and particularly of dominance with dominance interaction. Then quite small chance deviations in generation means can generate large estimates of genetic deviations. For satisfactory fitting of a trigenic model a more lateral design, that is one containing for example repeated backcrosses is required. In this connection an experiment by Hill (1966) with Nicotiana using repeated back-crosses, although producing some rather large interaction deviations, produced more believable results with a trigenic model. 
The improvement of fit with the digenic model with sex chromosome interactions was greater than that with the trigenic model (table 3), particularly in female tail length, a character not adequately fitted by any other combination of data model. Since some improvement of fit is suggested

TABLE 5

Estimates of the congenital parameters for the digenic model with interactions with sex chromosomes and without generati

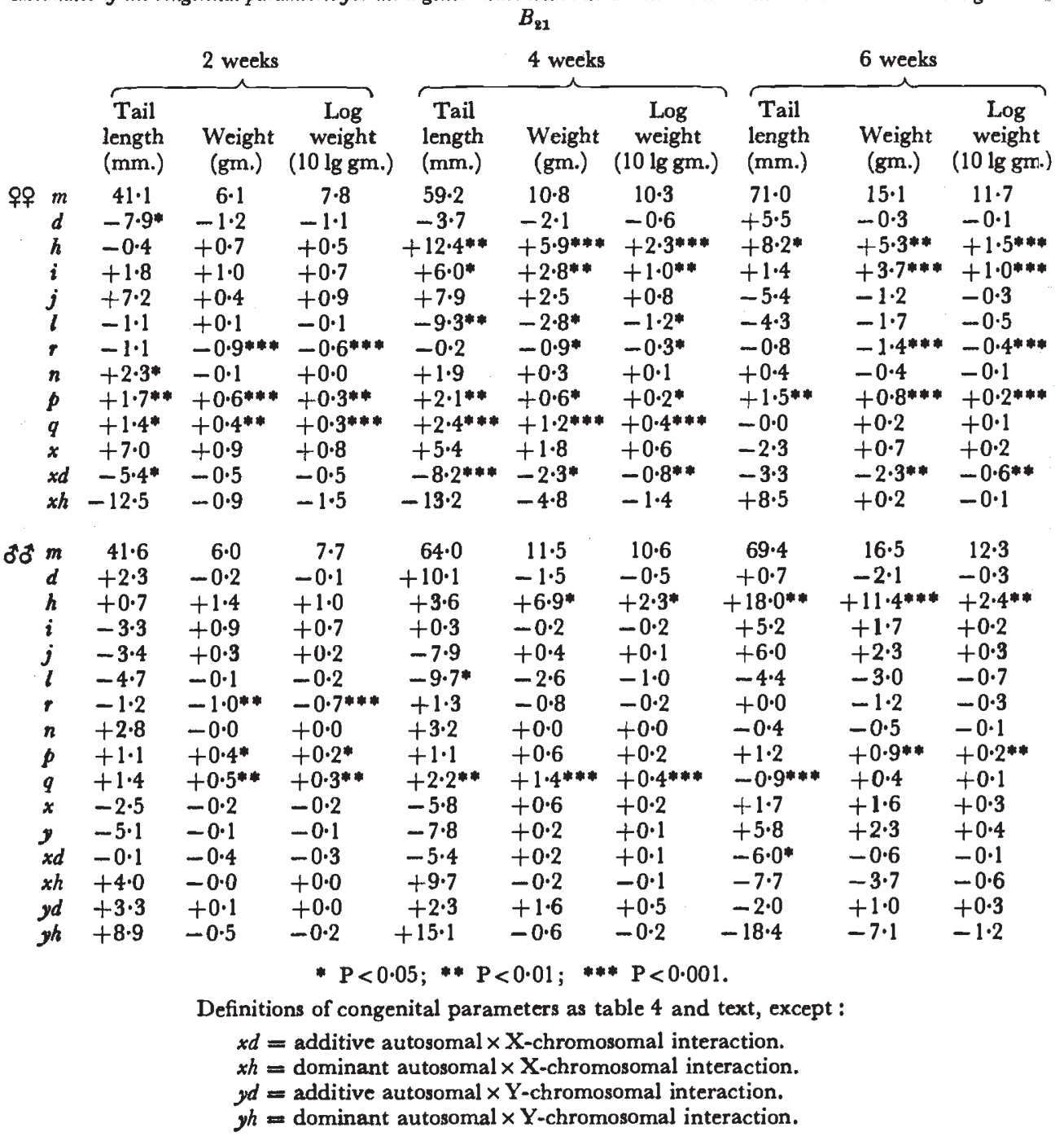

for male tail length and for female body weight at 4 and 6 weeks, the estimated parameters for all traits on this model are shown in table 5. The values of the parameters fitted for simpler genetic effects depend, of course, on the between-locus interactions contained in the model, so that it will be necessary to discuss the appropriateness of each model to each trait before choosing between the estimates in tables 4 and 5 . 
But before considering the significance of these results two points from the preliminary analysis must be recalled. Firstly, since the additive and logarithmic scales give closely similar results in the body weight analyis the argument that neither scale would be fully adequate is unimportant. Secondly, since there was evidence of genetic dispersion in the 2-week measurements, the additive and additive interaction parameters at that age will be inaccurate: " $d$ ", " $i$ " and " $x d$ " should have more positive values and " $j$ " more negative values than those shown. In the case of female body weights, the two-week measurements were also not fitted by any of the models tried, so that here we have effectively no valid estimates. In measurements on 6-week males the fit is less incomplete but still not perfect in any model; here the failure of fit stems from the unexplained smallness of animals of generation $\mathrm{B}_{12} \mathrm{~S}\left(A\right.$ 우 $\times\left(C B A \mathcal{O}^{\top} \times A\right.$ 우 $)$ 우 sibmated $)$.

There are three ways in which we can judge whether the data for the various traits are fitted adequately by the digenic model or better by the digenic model with sex-chromosome interaction. Firstly we may simply note from the significance levels in table 3 which traits are better fitted by the sex interaction model; the improvements by this criterion are all the tail length measurements except those on 2- and 6-week-old males, and none of the body weight measurements. Secondly, following Hill (1966), we may subtract the values of $\chi^{2}$ in rows 11 and 12 of table 3 from those in rows 7 and 8 , to see whether there is a significant improvement of fit with the addition of parameters for sex-chromosomal interaction; by this criterion all the female traits show significant improvement except 2-week weight and $\log$ weight and 6-week tail length which is on the margin of significance, but none of the male traits are significantly improved except 4- and 6-week tail lengths. Finally we may see in which traits significant estimated parameters for sex-chromosomal interactions were found; from table 5 it is seen that " $x d$ " is significant in all female traits except 2 -week weight and log weight and 6 -week tail length in which it was $1.78 \times$ its standard error, but in male traits only " $x d$ " in 6-week tail length is significant although in 4-week tail length it was $1.79 \times$ its standard error. Taking the three criteria together, it seems clear that the female data are best fitted by the model including sex-chromosomal interactions except in the case of 2-week weight and log weight for which no model is satisfactory, but that the male data are best fitted by the single digenic model except that for 4- and 6-week tail length the more complex model is preferable.

In assessing which parameters are truly significant, it is now necessary to choose between tables 4 and 5 according to which model is appropriate to the trait, to recall which values in the 2-week traits are liable to distortion due to dispersion, and further to refer back to table 3 for the overall confidence which may be placed on each set of figures. Accordingly table 6 has been drawn up on these bases. Estimates of parameters are entered in table 6 if they were significant in the appropriate model, excluding negative values of " $d$ " and " $x d$ " from 2-week measurements, and provided that where there remained a significant residual $\chi^{2}$ after fitting the significance of the value for the parameter exceeded that of the $\chi^{2}$. In preparing the table it was found that the direction and level of significance of the parameters for weight and $\log$ weight were identical except for 6-week-old males. Therefore the log weight values have been omitted in the other cases as a further simplification. 
We can reach some general conclusions from the values in table 6 and see whether they are confirmed by the fuller results of tables 4 and 5 as appropriate. All of the autosomal and maternal effects are significant in one or other of the traits, but only " $y$ " and " $x d$ " of the sex-chromosomal direct and interaction effects appear in table 6 . The negative effect of " $y$ " is confirmed in the body weight estimates of table 4, significantly at 6-weeks, and in the 4-week but not in the 6-week tail lengths in table 5. Similarly

TABLE 6

Extract from tables 4 and 5 to show effects accepted as significant and true $\dagger$

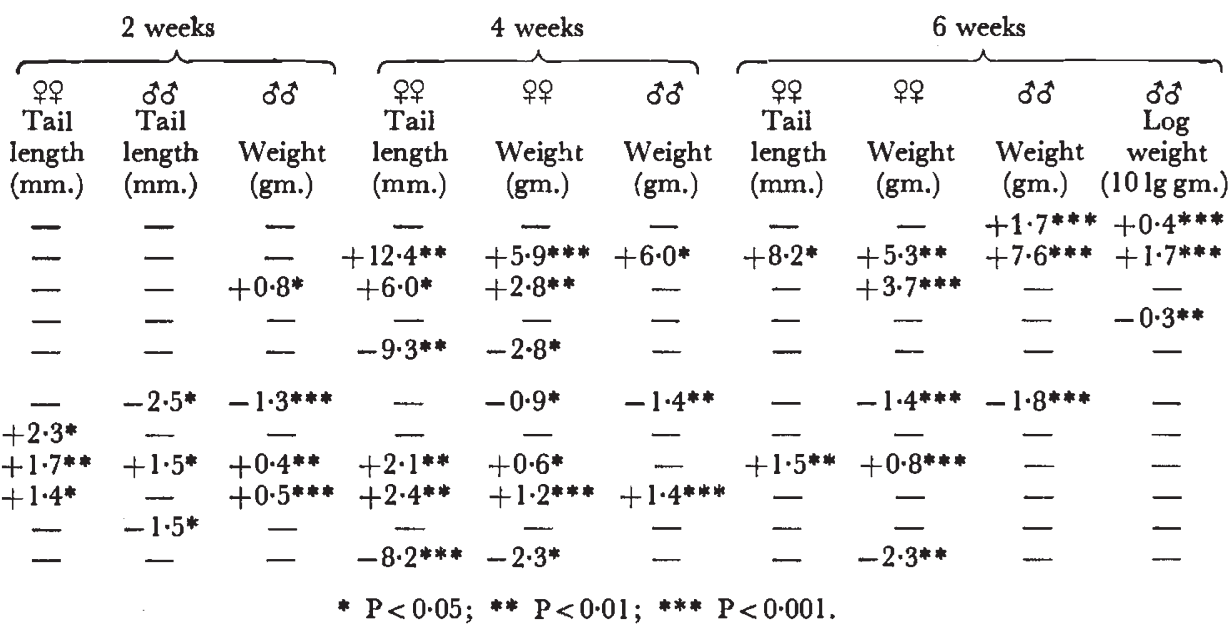

For definitions of parameters see text p. 560 and table 5.

$\dagger$ For explanation of criteria, see text, p. 565.

" $x d "$ is negative for all traits for which its estimation is appropriate, and significant in most of them. The value of " $x$ " is positive in all female traits except one (table 5) and before sex-chromosomal interactions were introduced some of its estimates were highly significant. We cannot therefore dismiss the possibility of classical sex-linked effects on female body size. On the other hand, the effects of " $x$ " " $y h$ " and " $y d$ " in males and of " $x h$ " in both sexes show no evidence of significance or consistency.

Of the maternal effects the positive effect of $C B A$ dams (" $n$ ") seems restricted to 2-week or possibly 2- and 4-week tail lengths, but the remainder are consistent throughout the data. $A$ dams (" $r$ ") consistently suppress growth and both $\mathrm{F}_{1} \mathrm{~s}$ (" $p$ " and " $q$ ") enhance it, but with the difference that at 6 weeks the positive value of " $p$ " $\left(A Q \times C B A{ }^{*}\right.$ dam $)$ remains large and significant while that of " $q$ " for the reciprocal $\mathrm{F}_{1}$ dam is much reduced.

Among the autosomal genetic effects only " $i$ " (additive $\times$ additive deviation) is consistent in its effects. Taking the appropriate values from tables 4 and 5 , in only one case, male 2-week tail length, is there a negative value of " $i$ ", and of the other 15 positive values 9 are significant. The negative effect of " $j$ " (additive $\times$ dominance interaction) is restricted to the two measures of 6-week male body weight and may represent the occasional false "significant" effect expected in an analysis of this size. " $h$ " (dominance deviation) is significantly positive for all traits at 4 and 6 weeks of age 
and positive but not significant in 2-week males. " $l$ " (dominance $x$ dominance interaction) is significantly negative in all measures on 4-week females. Reference to table 5 shows that it is also significantly negative in 4-week male tail length and from both tables " $l$ " is negative to a lesser non-significant degree in the remaining 4 and 6-week traits. " $d$ " (additive deviation) is highly significantly positive in both measures of 6-week male body weight (table 6). In table 5 , the significant negative value of " $d$ " in 2-week female tail length is dismissed as due to dispersion, and the only remaining value of interest is the large but non-significant positive " $d$ " for female 6-week tail length.

\section{Discussion}

The maternal effects in the present analysis were important and persisted up to 6 weeks with little diminution. Similar persistence of maternal effects on body weight has been reported on a number of occasions, for example in several earlier experiments with mice reviewed by Roberts (1965). More recently Monteiro and Falconer (1966) found that maternal effects were the most important cause of variance in mouse body weight and increased up to 4 weeks of age, compensation being delayed to the period of growth from 5 to 8 weeks. In the rat Jinks and Broadhurst (1963) found that maternal effects were more important at 50 days of age than at weaning (21 days). In the chicken, where direct maternal influence ceases when the egg is laid, Cock and Morton (1963) found that none the less the pure-line maternal effect remained nearly constant, as a percentage of total body weight, throughout growth up to 20 weeks post-hatching. Thus the present results fit into a general pattern in which maternal effects on body size, far from ceasing with the break from the mother, persist at least throughout the acceleratory phase of growth. The present data also confirm the conclusion of Brumby (1960) that heterozygosity is more effective than mere size of dams in enhancing growth, so that it is the complete maternal environment that limits the first phase of growth. That this environment is most complex is demonstrated by the larger size of offspring from the $\left(A q \times C B A \sigma^{\top}\right) \mathrm{F}_{1}$ than from the reciprocal $F_{1}$ dams. This is not an effect of the size of the dam, as the $\left(C B A+{ }^{\circ} \times A_{\circlearrowleft}\right) \mathrm{F}_{1} \mathrm{~s}$ were slightly the larger. Nor is it simply a cytoplasmic effect with the smaller strain $(A)$ exerting a plus influence on size, and vice versa, because in the trials of various models described earlier, replacement of two parameters for each $F_{1}$ maternal effect by a single parameter for the maternal effect and a new one for a cytoplasmic effect of this kind produced a worse fit to the data. We are forced to postulate some form of interaction between the $A$ strain cytoplasm and the $\mathrm{F}_{1}$ genotype. Cytoplasmic effects have been previously demonstrated unequivocally by Brumby (1960), using ovum transplantation. The body weights of the reciprocal crosses of two Peromyscus species investigated by Dawson (1965) could not be explained except as interaction between a " maternal element" and polygenic factors, but since his experiment was not designed to distinguish between cytoplasmic factors and the general maternal environment, the latter provides a simpler explanation of his results.

There is very little evidence in the literature for sex-linked effects on mouse body size, and they are often assumed negligible (e.g. Eisen and Legates, 1966). However, the reason for the lack of evidence is that few 
published analyses permit the unequivocal demonstration of sex-linkage. Garmon (1963) found very significant reciprocal differences remaining between crosses after fitting for maternal effects, and attributed them to sex-linkage. But the work of Brumby (1960) which could clearly have demonstrated sex-linkage showed no evidence of it. In the present analysis, the effects of Y-linkage were significant and not unimportant throughout the data. The $\mathrm{Y}$ chromosome of the larger $C B A$ line reduced size relative to the effect of the $A$ line chromosome. No direct effect of the $\mathrm{X}$ chromosome could be demonstrated, but the results were suggestive of an effect whereby the $C B A \mathrm{X}$ chromosome produced larger females and that of the $A$ line smaller females. The main contribution of the $\mathrm{X}$ chromosomes was found to lie in their interaction with the autosomes, shown by the consistent negative value of " $x d$ ". This means that the $\mathrm{X}$-linked and autosomal genes within each inbred line interact so as to reduce growth. The present findings show that the maintenance of sex differential in body weight is much more complicated than a simple enhancement of the normal mode of action of the autosomal genes, as was suggested by Butler (1952) and used by Morton and O'Donald (1962) as the rationale for their scaling method. This complexity accounts for the failure here of the scaling method that we proposed. The value for " $x$ ", " $y$ " and " $d$ " in tables 4 and 5 suggest that the sex differential is achieved, at least in part, by different mechanisms in the two inbred lines. Autosomal genes for large size derived from $C B A$ tend to be suppressed in females, while it is the $\mathrm{X}$ and $\mathrm{Y}$ chromosomes from $A$ that contribute most to the difference in size between the sexes. A sex differential in body size, particularly the male positive differential in mammals, is so regular a phenomenon that one must assume that it has selective advantage, and hence that genes whose actions are modifiable by the sexual environment will accumulate in evolution. But it is equally self-evident that there must be some optimal value for the differential beyond which it will become disadvantageous. From the $\mathrm{F}_{2}$ of the cross of $A$ and $C B A$, selection could greatly increase the sex differential by selecting $C B A$ autosomes and $A$ sex chromosomes with a concomitant increase in overall size, or decrease it by the exact opposite. Korkman (1957) demonstrated that sex differential could be increased or decreased by artificial selection in a population derived from wild mice. He found no important overall changes in body weight, but he showed that the real effect of his selection had been an alteration in the growth rates of males between 30 and 60 days, so that in this respect the genes were similar to the autosomal additive effects of $C B A$ genes. The fact that male growth rates were more easily affected than female is significant to the remainder of this discussion.

Two overall impressions from the estimates of genetic effects require discussion, the extent and importance of non-additive gene action and possible trends of the genetic effects with age.

There exists a general impression that non-additive effects are of little importance in the inheritance of mouse body size (e.g. Roberts, 1965). This impression rests heavily on the work of Miller, Legates and Cockerham (1963), which has also been quoted as a justification for ignoring nonadditive effects in analytical design (Monteiro and Falconer, 1966). Yet Miller et al. found considerable negative components of variance attributable to non-additive effects, which they interpreted conventionally as representing zero effects with large sampling errors. The assumption of large errors 
implies that the form of analysis was unable to assess accurately the contribution from genic interaction or to exclude positive values as great as the negative ones found. Miller et al. were themselves aware of this problem, and considered alternative forms of variance analysis, but concluded that these could not distinguish between genic and genotype-environment interaction.

In analyses involving comparisons of the means of inbred or selected lines and crosses between them, either positive or negative heterosis for body weight has often been found (Roberts, 1965), although the positive form is the more common (Roberts, 1967). In the present data the means of the two $\mathrm{F}_{1}$ s comfortably exceeded the means of the two inbred lines in all measurements at 4 and 6 weeks. But from the results of the analyses the way in which this heterosis is built up from simpler genetic effects is complex. The consistent positive value of " $i$ " (additive $\times$ additive interaction) will oppose the positive heterosis. This positive " $i$ " no doubt represents the response of the two inbred lines to selection between sib pairs during their development. The inbred lines will have developed balanced pairs of genes which resist the reduction of body size due to inbreeding depression. To the extent that gene pairs can resist inbreeding depression they naturally reduce heterosis. In the analyses of male body weight, in which there is no evidence of autosomal $\times$ sex-linked interaction, the heterosis remains positive because the positive value of " $h$ " (dominance deviation) exceeds the negative value of " $l$ " (dominance $\times$ dominance interaction) by comfortably more than the value of " $i$ ". In the remaining 4- and 6-week traits, the positive " $h$ " again generally exceeds the negative " $l$ ", but this alone would not be sufficient to maintain positive heterosis if it were not that the positive value of " $i$ " was offset by a negative " $x d$ " (autosomal additive $x$ $\mathrm{X}$-linked additive interaction). If such a complex set of balances exist generally in mouse body size inheritance, small changes in the relative size of the participating effects could explain the variety of forms of heterosis noted by Roberts (1965).

The effect in a population of the opposition of " $h$ " and " $l$ " is to minimise the difference between those animals heterozygous at few loci and those heterozygous at many. Thus this opposition will tend not to generate variance within a single outbred population. None the less it might be possible, in these circumstances, to demonstrate non-additive genetic variance in crosses between lines. Jinks and Broadhurst (1963), applying sophisticated variance analyses to a diallel cross among inbred lines of rats, demonstrated dominance, but not epistasis. However, their method for detecting epistasis, the $W r / V r$ regression, is particularly insensitive to the detection of duplicate-type interaction (Hayman, 1954), which for two loci means the ability of a single dominant gene at either locus to achieve the effect. This type of interaction is represented in the terms of this paper by opposing values of " $h$ " and " $l$ ". Such a form of gene action, which generates little detectable variance even in refined analysis, clearly belongs to that substructure of the genome concerned with the maintenance of homeostasis (Lerner, 1954).

Changes of genetic effects with age have been noted before. In mice, Young and Legates (1965) found negative genetic correlations between earlier and later growth, from which they concluded either that different genes were concerned or that the same genes were showing opposite effects 
in the two periods; and Monteiro and Falconer (1966) showed that additive genetic variance increased with age both absolutely and as a proportion of phenotypic variance. Of particular interest is the rat experiment of Jinks and Broadhurst (1963). They found differences both with sex and age, in particular that the dominance ratio (ratio of variance from dominant effects to that from additive effects) was greater at 50 days than at 100 and greater in females than in males. A similar difference between sexes was found by Kidwell and Nash (1964) when reviewing data from three species for evidence of sex $\times$ heterosis interaction. They concluded that where demonstrable the interaction was usually due to greater heterosis in females than in males, and that it probably involved not homogametic (X-chromosomal) heterosis itself, but an interaction either between the sex-determined environment and the genotype or between sex-linked and autosomal gene loci.

It will be recalled from the Results section that, in contrast to " $i$ ", " $y$ ", and the maternal effects which were generally consistent throughout the data, " $d "$ (additive deviation), " $h$ " (dominance deviation) and " $l$ " (dominance $x$ dominance interaction) showed different effects in different parts of the data. They were all effectively absent in the 2-week data (if we accept that negative values of " $d$ " are artefacts due to dispersion). " $h$ " is large, positive and mostly highly significant throughout the 4- and 6 -week data (table 6). " $l$ " is large, negative and significant in 4-week females, but is reduced in size and loses significance at 6 weeks. An identical trend but with less significance can also be seen in the male data. " $d$ " is positive and highly significant for 6-week male body weight only, although there is also the suggestion that it is large and positive, but not significant in female 6-week tail length. These trends could be explained, and related to the earlier work cited, by postulating that one general form of inheritance succeeds another in the course of maturation, but is delayed in females relative to males. The young female form consists in duplicate-type interaction of heterozygotes, demonstrated by values of " $h$ " and " $l$ " which are nearly equal but of opposite sign, with no detectable additive effects. This is replaced by an adult male form in which the genes for large size are simply dominant to those for small size.

A certain care is needed in interpreting opposing values of " $h$ " and " $l$ " because their estimates tend to be negatively correlated. Where, as in 4week female tail length, the estimates of " $h$ " and " $l$ " are both highly significant this is unimportant, but non-significant negative values of " $l$ " could have been generated in part by the significant positive values of " $h$ " and vice versa in other characters. But the degree of negative correlation depends on the experimental design (Hayman, 1958) and is therefore the same at all ages. Hence the trend whereby the young female pattern of " $+h-l "$ is replaced by an adult male pattern of " $+d+h$ " cannot be due to the correlation of the estimaters.

The juvenile female form may be thought of as overdominance for intermediacy; such as was deduced by Jinks and Broadhurst (1963) for the genetical control of birthweight in rats. As has been mentioned, this form of gene action even when of considerable importance yields little detectable variance, and may be considered as primarily a homeostatic effect. In the adult male form additive (" $d$ ") effects are more important (Monteiro and Falconer, 1966) and the genes for large size show dominance (Roberts, 1967), which is often complete (" $d "=" h ")$, so that the dominance ratio 
is close to unity (Jinks and Broadhurst, 1963). This form of inheritance clearly generates variance, and it is notable that males show greater variance of body measurement than females (Jinks and Broadhurst, 1963) whether this is measured on a additive or logarithmic scale or whether male or female is the heterogametic sex (Morton, 1963). The clear corollary of this conclusion is that accurate homeostatic control of body size is more important in juveniles than in adults and in females than in males. Considering the effects of litter competition, and the different reproductive roles of female and male, this corollary does not appear unreasonable. A similar contrast has been drawn by Kearsey and Kojima (1967), working with Drosophila. They noted that mature body weight, which they presumed to be under the control of stabilising selection, showed additive gene action, but that hatchability, under directional selection, showed opposing dominance and dominance interaction.

The opposition of " $h$ " and " $l$ ", as found in the young female type, would be expected in characters which fit the models for the inheritance of fitness proposed by Sved et al. (1967) and King (1967). In these models the setting of an upper limit to fitness together with heterozygote advantage implies that dominance interaction should oppose dominance. This conclusion implies an extension of the hypothesis of Robertson (1955) that the more closely a character is related to fitness, the less additive and the more dominant or overdominant variation it will exhibit. If the fitness of the individual depends on homeostatic control of the related character the opposition of dominance and dominance interaction will be expected. But then the resultant measurable variability, like the degree of variability in fitness exhibited among the more probable genotypes in the Sved-King model, will be small.

It may be reversing the logical order to describe the " $h$ " " $l$ " opposition as a consequence of the Sved-King model. If this model represents the true situation, it will be the result of natural selection acting on genetic effects. This may be seen by considering how selection could act to increase the fitness of the homozygotes of an initially independent polymorphism. If the effect of selection on some locus capable of interacting with the polymorphic locus is to replace one allele with another, the polymorphism will continue to appear independent when observed at any one time. But to the extent that it is the heterozygotes at further loci which enhance the fitness of the homozygotes at the first locus, we shall observe a situation where only multiple homozygotes are deleterious and all heterozygotes are generally fit. Then, in a character closely related to fitness, single and double heterozygotes will have equal value and dominance $\times$ dominance interaction will appear opposed to dominance. The combination of components of fitness inherited in this manner would give a distribution of total fitness like that of the SvedKing model.

\section{SUMMARY}

1. Gene action and interaction in the control of body weight and tail length of mice at 2, 4 and 6 weeks of age are investigated in the $\mathrm{A} / \mathrm{Cag}$ and $C B A / C a g$ inbred lines and crosses between them up to the fourth filial and sibbed back-cross generations, involving measurements on more than 3500 mice. 
2. Preliminary analyses concern correct scaling and the degree of association or dispersion of the size of genes in the $A$ and $C B A$ lines.

3. It is concluded that the additive scale is appropriate for the analysis of tail length, but that both additive and logarithmic scales should be used for body weight, as no one scale is fully adequate.

4. At 4 and 6 weeks the size genes are fully associated in the two lines, but at 2 weeks there is evidence of dispersion, implying caution in interpreting the estimates of additive and additive interaction effects from the main analysis.

5. The main analysis is by least squares equation of the generation means to the expected proportions of parameters for the different forms of gene action, yielding estimates of additive, dominance and 2-locus interaction effects, and of sex-linkage and the maternal effects of the parental and reciprocal $\mathrm{F}_{1}$ dams.

6. For the female data and for 4- and 6-week tail length in males it was necessary to include 2-locus interactions between sex-linked and autosomal loci. There was no evidence of these effects in the remaining male data.

7. Important maternal effects persist up to 6 weeks of age. In addition to the known effects of heterozygosity and maternal size, there is evidence for cytoplasmic interaction. Y-linked inheritance is demonstrated, and $\mathrm{X}$-linked suggested.

8. The sex-differential in body size appears to be maintained largely by sex-linkage in the $A$ line, but by autosomes which increase size only in males in $C B A$.

9. The main autosomal effects change with age and with sex. These changes may be explained as the replacement of the early duplicate interaction type of inheritance of opposing dominance and dominance $\times$ dominance interaction by a simpler form of inheritance in which genes dominant for large size act independently at each locus.

10. This process starts earlier and becomes more complete in males than in females, the difference being related to the greater need for homeostatic control in juvenile than in adults and in females than in males.

11. It is suggested that natural selection to maintain homeostatis will lead both to duplicate-type interaction and to the non-linear relationship between fitness and gene substitution which is the basis of several recent theoretical papers.

Acknowledgments. - I wish to thank the Director of the Cambridge University Mathematical Laboratory for use of the Titan computer; Mr Robert Marrs for aid with programming; Professor J. L. Jinks for a number of useful suggestions; and particularly Dr D. G. Gilmour for his help and encouragement.

\section{REFERENCES}

Biggers, J. D., AShoub, M. R., MClaren, A., AND michie, D. 1958. The growth and development of mice in three climatic environments. F. Expl Biol., 35, 144-155.

BREESE, E. L., AND MATHER, K. 1960. The organisation of polygenic activity within a chromosome in Drosophilia. Heredity, 14, 375-399.

BRUMBY, P. J. 1960. The influence of the maternal environment on growth in mice. Heredity, 14, 1-18.

BUTLER, L. 1952. A study of size inheritance in the house mouse. II. Analysis of four preliminary crosses. Canad. 7. Zool., 30, 154-171.

CARMON, J. L. 1963. Heterosis, combining ability, and maternal effects in mice. 7. Genet., $58,225-231$. 
CAVALLI, L. L. 1952. An analysis of linkage in quantitative inheritance. Quantitative Inheritance. H.M.S.O., London, pp. 135-144.

CHAI, c. K. 1956. Analysis of quantitative inheritance of body size in mice. II. Gene action and segregation. Genetics, 41, 165-178.

CHAI, C. K. 1957. Analysis of quantitative inheritance of body size in mice. III. Dominance. Genetics, 42, 601-607.

COCK, A. G., AND MORTON, J. R. 1963. Maternal and sex-linked effects on size and conformation in domestic fowl. Heredity, 18, 337-350.

Dawson, w. D. 1965. Fertility and size inheritance in a Peromyscus species cross. Evolution, 19, 44-55.

EISEN, E. J., AND LEGATES, J. E. 1966. Genotype-sex interaction and the genetic correlation between the sexes for body weight in Mus musculus. Genetics, 54, 611-623.

Falconer, D. s. 1947. Milk production in mice. F. Agric. Sci., 37, 224-235.

FALCONER, D. S. 1953. Selection for large and small size in mice. $\mathcal{J}$. Genet., 51, 470 498.

Gilbert, N. 1961. Polygene analysis. Genet. Res., 2, 96-105.

GILMOUR, D. G., AND MORTON, J. R. 1970. Association of genetic polymorphisms with embryonic mortality in the chicken. III. Egg white proteins and the pure and crossbred progeny of two populations. Theoret. Appl. Genet. (in press).

HARRISON, G. A., MORTON, R. J., AND WIENER, J. s. 1959. The growth in weight and tail length of inbred and hybrid mice reared at two different temperatures. Phil. Trans. (b), $242,479-516$.

haYman, B. I. 1954. The theory and analysis of diallel crosses. Genetics, 39, 789-809.

HAYMAN, B. I. 1958. The separation of epistatic from additive and dominance variation in generation means. Heredity, 12, 371-390.

HILL, J. 1966. Recurrent backcrossing in the study of quantitative inheritance. Heredity, $21,85-120$.

JINKS, J. L., AND BROADHURST, P. L. 1963. Diallel analysis of litter size and body weight in rats. Heredity, 18, 319-336.

JINKs, J. L., AND JONES, R. M. 1958. Estimation of components of heterosis. Genetics, 43, 223-234.

Jinks, J. L., AND PERKINS, J. M. 1969. The detection of linked epistatic genes for a metrical trait. Heredity, 23, 465-475.

KEARSEY, M. J., AND KOJIMA, K.-I. 1967. The genetic architecture of body weight and egg hatchability in Drosophila melanogaster. Genetics, 56, 24-37.

KIDWELL, J. F., AND NASH, D. J. 1964. An examination of homogametic heterosis in three species of laboratory animals. Canad. 7. Genet. Cytol., 6, 207-214.

KING, J. L. 1967. Continuously distributed factors affecting fitness. Genetics, 55, 483-492.

KORKMAN, N. 1957. Selection with regard to the sex difference of body weight in mice. Hereditas, 43, 665-678.

LERNER, I. M. 1954. Genetic Homeostasis. Oliver and Boyd, Edinburgh and London.

LYON, M. F. 1962. Sex chromation and gene action in the mammalian X-chromosome. Amer. 7. Hum. Genet., 14, 135-148.

MATHER, K. 1949. Biometrical Genetics. Methuen and Co. Ltd., London.

MATHER, K. 1966. Selection and Variation. Proc. Roy. Soc, B., 164, 341-349.

MiLleR, R. H., LEGATES, J. E., AND COCKERHAM, c. c. 1963. Estimation of non-additive hereditary variance in traits of mice. Genetics, 48, 177-188.

MONTEIRO, L. S., AND FALCONER, D. s. 1966. Compensatory growth and sexual maturity in mice. Anim. Prod. 8, 179-192.

MORTON, J. R. 1963. A study of the inheritance in the mouse and the chicken, of certain metric and biochemical traits. Ph.D. thesis, Cambridge University Library.

MORTON, J. R. 1964. Gene action and interaction in the control of metric traits in chickens and mice. Heredity (Abstr.), 19, 171.

MORTON, J. R., GILMOUR, D. G., MCDERMID, E. M., AND OGDEN, A. L. 1965. Association of bloodgroup and protein polymorphisms with embryonic mortality in the chicken. Genetics, 51, 97-107.

MORTON, J. R., AND O'DONALD, P. 1962. Analysis of gene action from inbred line crosses in nonself-fertilising organisms. Heredity, 17, 553-559.

ROBERTS, R. C. 1965. Some contributions of the laboratory mouse to animal breeding research. Part I. Anim. Br. Abstr., 33, 339-353.

ROBERTs, R. C. 1967. The limits to artificial selection for body weight in the mouse. III. Selection from crosses between previously selected lines. Genet. Res., 9, 73-85. 
ROBERTSON, A. 1955. Selection in animals: Synthesis. Cold Spr. Harb. Symp. Quant. Biol., 20, 225-229.

SMITH, J. MAYNARD. 1968. "Haldane's Dilemma" and the rate of evolution. Nature, 219, $1114-1116$.

STAATs, J. 1968. Standardized nomenclature for inbred strains of mice; fourth listing. Cancer Res., 28, 391-420.

SVED, J. A., REED, T. E., AND BODMER, W. F. 1967. The number of balanced polymorphisms that can be maintained in a natural population. Genetics, 55, 469-481.

williams, w. 1959. Heterosis and the genetics of complex characters. Nature, 184, 527-530. WRIGHT, s. 1952. The genetics of quantitative variability. In Quantitative Inheritance (Agricultural Research Council). H.M.S.O., London.

younG, C. W., AND LEGATES, J. E. 1965. Genetic, phenotypic, and maternal interrelationships of the growth in mice. Genetics, 52, 563-576. 